\title{
Thermoluminescence properties of $\gamma$-irradiated Bi doped BaS nanostructures
}

\author{
SURENDER SINGH*, ANKUSH VIJ, S P LOCHAB ${ }^{\dagger}$, RAVI KUMAR ${ }^{\dagger \dagger}$ and \\ NAFA SINGH \\ Department of Physics, Kurukshetra University, Kurukshetra 136 119, India \\ ${ }^{\dagger}$ Inter University Accelerator Center, Aruna Asaf Ali Marg, Post Box 10502, New Delhi 110 067, India \\ ${ }^{\dagger \dagger}$ Centre for Material Research, National institute of Technology Hamirpur, India
}

MS received 1 April 2010; revised 21 August 2010

\begin{abstract}
Bismuth doped barium sulphide nanocrystallities were prepared and characterized by XRD. Thermoluminescence (TL) studies of these samples after exposure to gamma radiation were carried out. The TL glow curve of the phosphors have two peaks at $403 \mathrm{~K}$ and $658 \mathrm{~K}$ while in their bulk counterparts these peaks were reported at $486 \mathrm{~K}$ and $570 \mathrm{~K}$ (Rao 1986). We noted that TL intensity increases with gamma exposure time in the range $30 \mathrm{~min}-41 \mathrm{~h}$ which may be explained on the basis of track interaction model (TIM) and a high surface to volume ratio for the nanostructures. The kinetic parameters at various heating rates namely activation energy (E), order of kinetics (b) and frequency factor (s) of BaS : Bi (0.4 mol\%) sample was determined using Chen's method. The deconvolution of curve was done using the GCD function suggested by Kitis. The effect of different heating rates and different amount of dose has also been discussed.
\end{abstract}

Keywords. Nanostructures; X-ray diffraction; luminescence.

\section{Introduction}

Thermoluminescence (TL) is a simple technique for obtaining information on the distribution of artificially created or naturally occurring point defects. The light emitted during TL process is plotted as intensity vs temperature when the charge carriers are released. The charge carriers are trapped for an extended period of time waiting to acquire enough energy to increase the probability to escape, producing electromagnetic radiation. The stimulation energy to release these electrons is usually thermal and the phenomenon is called thermoluminescence, widely used to measure different kinds of radiation doses. Alkaline earth sulphides (AES) are well known from their luminescent properties. Ionising radiations like UV, X-ray, gamma ray electrons, etc produce localized levels in the activated phosphors and the energy storage by such traps is studied by using the conventational TL glow curve method. Nanomaterials are defined as those materials whose length scale lies within the nanometric range, i.e. in the range between one to a hundred nanometers. Within this length scale, the properties of matter are considerably different from the individual atoms, molecules and bulk materials. The physical, chemical, electrical and optical properties of these materials are size- and shape-dependent and they often exhibit important diffe-

*Author for correspondence (surendernain07@gmail.com) rences in the bulk properties. Lochab et al (2006) and Sahare (2007) showed that in nanomaterials the peak temperature shifts towards higher side and they show a large linear range over very high doses as compared to their bulk counterparts. The traps created in nanomaterials are deeper than in bulk materials. The different behaviours of nanomaterials can be explained on the basis of quantum size effect and large surface to volume ratio. We have already studied the TL properties of cerium doped SrS from UV radiation (Vij et al 2009a, b). This work promotes us to work on other members of AES.

This paper reports the response of TL glow curves of $\mathrm{BaS}$ : Bi nanocrystalline after exposure to gamma ray at various doses. The effect of different doses and different heating rates on glow curves has been determined and discussed. Before using a phosphor for radiation dosimetry, a prior knowledge of traps and kinetic parameters of a sample is desirable. We have used Chen's peak method and glow curve deconvolution function to analyse the TL glow curve and calculated the trapping parameters such as activation energy $(E)$, order of kinetics $(b)$ and frequency factor(s) for Bi doped BaS nanostructures.

\section{Experimental}

Barium sulphide nanostructures were prepared by solid state diffusion method. Bismuth $(0 \cdot 4 \mathrm{~mol} \%)$ was used as 
a dopant. The details of phosphors preparation can be found elsewhere (Singh et al 2010). XRD of the given samples was obtained using a model D8-Advance of Bruker (Germany), of CuK-alpha radiation of $8.04 \mathrm{keV}$ and wavelength $1.54 \AA$. The applied voltage was $40 \mathrm{kV}$ and current was $25 \mathrm{~mA}$. Phosphors were exposed to $\gamma$ rays from a $\mathrm{Cs}^{137}$ source for various doses at room temperature. One hour exposure from this source corresponds to $115 \mathrm{mR}$. TL glow curves were recorded at heating rate of $2 \mathrm{Ks}^{-1}, 5 \mathrm{Ks}^{-1}$ and $10 \mathrm{Ks}^{-1}$ on a Harshaw TLD reader (Model 3500) having a standard clear glass filter (a cleanout/neutral density filter to neutralize the nonlinear response of the PMT), taking $5 \mathrm{mg}$ of sample each time.

\section{Result and discussions}

\section{$3.1 X R D$}

The XRD pattern of all the samples was nearly the same and hence only one pattern is shown in figure 1. The average size of the nanoparticles was estimated from the line broadening of the XRD peaks and Scherer's equation (Scherer 1918)

$$
d=\frac{0 \cdot 89 \lambda}{\beta \cos \theta_{\mathrm{B}}},
$$

where $d$ is the average diameter of the nanoparticles, $\lambda$ is the wavelength of $\mathrm{CuK} \alpha(1.54 \AA)$ radiation, $\beta$ (in radians) is full width at half maxima (FWHM) and $\theta_{\mathrm{B}}$ is the Bragg angle. The structure of the host material is cubic (rock salt). The average particle size was $35 \mathrm{~nm}$. The pattern was compared with JCPD card no. 75-0896.

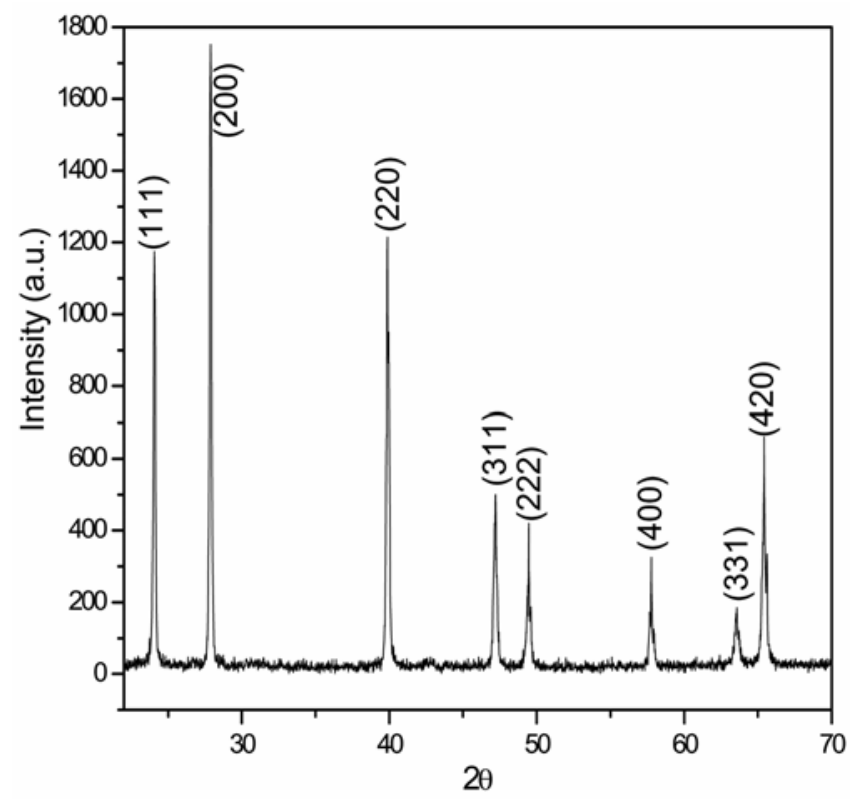

Figure 1. XRD pattern of $\mathrm{BaS}: \mathrm{Bi}(0.4 \mathrm{~mol} \%)$ nanocrystalline.

\subsection{Thermoluminescence responses}

Figure 2 shows a typical TL glow curve for the nanocrystalline $\mathrm{BaS}: \mathrm{Bi}$ exposed to various doses of gamma rays from a $\mathrm{Cs}^{137}$ source. The nanomaterials powder has two TL glow peaks at $403 \mathrm{~K}$ and $658 \mathrm{~K}$. The peak at $403 \mathrm{~K}$ is very broad which may consists of several overlapping peaks. Later these peaks were separated by thermal cleaning method (Yazici and Haciibrah 2001). The glow curve structure and the peak shape does not change for the exposure dose. In figure 3 , the TL intensity for peak at $403 \mathrm{~K}$ is plotted as a function of exposure time where a more or less linear response is observed for exposure time of $41 \mathrm{~h}$. This linearity over a wide range of doses

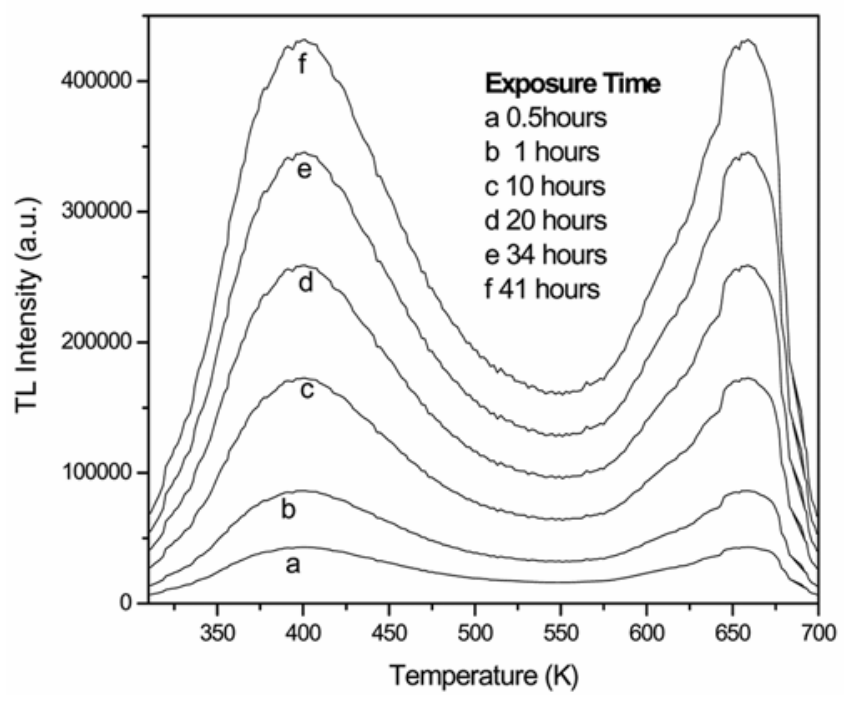

Figure 2. TL glow curves of $\mathrm{BaS}: \mathrm{Bi}(0.4 \mathrm{~mol} \%)$ after different exposure times at a heating rate of $2 \mathrm{Ks}^{-1}$.

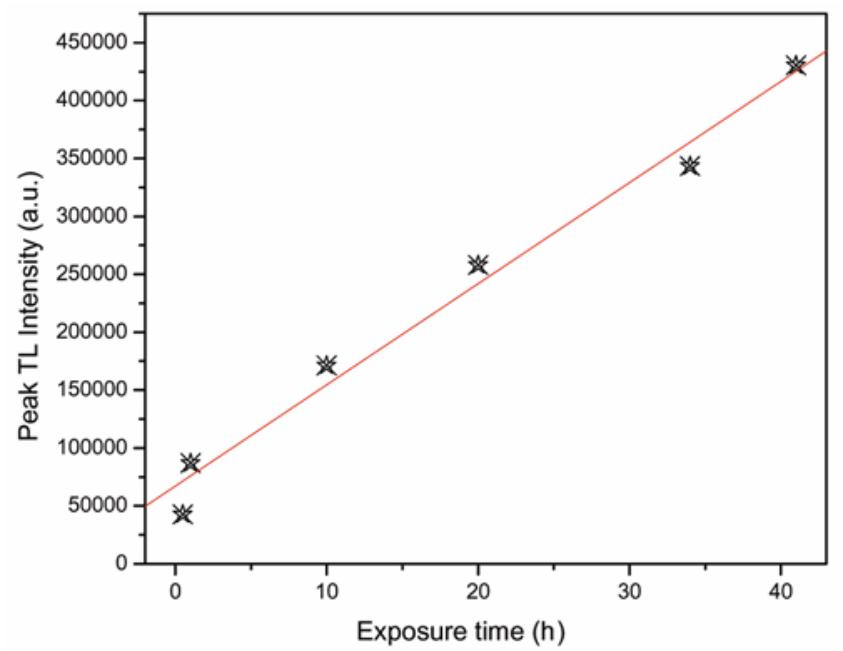

Figure 3. The peak TL intensity of $\mathrm{BaS}: \mathrm{Bi}$ as a function of exposure time at the heating rate of $2 \mathrm{Ks}^{-1}$. 
may be explained on the basis of track interaction model (Mahajna and Horowtz 1997; Horowtz et al 2001). According to this model the number of generated traps due to irradiation depends upon both the length of tracks in matrix and area of cross-section of tracks. In case of nanomaterials there still exist some particles that would have missed - due to very tiny size - while being targeted by high energy radiation. Thus on increasing the dose these nanoparticles which had earlier been left out from the radiation now generate trapping and luminescent centers. So we do not get saturation in nanomaterials even at higher doses. This may also be explained on basis of large surface to volume ratio which results in a higher surface barrier energy for nanoparticles. Thus on increasing the doses, the energy density crosses the barrier and a large number of defects produced in the nanomaterials ultimately keep on increasing with the dose till saturation is achieved.

\subsection{Effect of various heating rates on TL response of $B a S: B i$}

Figure 4 shows the TL glow curve of $\mathrm{BaS}: \mathrm{Bi}$ at various heating rates from $2 \mathrm{~K} / \mathrm{s}$ to $10 \mathrm{~K} / \mathrm{s}$. From these curves we found that the maximum glow peak temperature $\left(T_{\mathrm{m}}\right)$ shifted to higher temperatures and the TL intensity decrease as heating rates increases. The first peak in TL glow curve changes from $403 \mathrm{~K}$ to $427 \mathrm{~K}$ as heating rates change from $2 \mathrm{Ks}^{-1}$ to $10 \mathrm{Ks}^{-1}$. The reduction in TL intensity is attributed to thermal quenching effect (Akselrod et al 1998), the efficiency of which increases as temperature increases.

\subsection{Analysis of TL glow curve and calculations of trapping parameters}

The determination of kinetic parameters has been an active area of research and various techniques have been developed to derive these parameters from the glow curve. However we apply peak shape method to the whole experimental glow curve and evaluate the parameters which define as

$$
\omega=T_{2}-T_{1}, \delta=T_{2}-T_{\mathrm{m}} \text { and } \tau=T_{\mathrm{m}}-T_{1},
$$

where $T_{\mathrm{m}}$ is the maximum temperature and $T_{1}$ and $T_{2}$ are low and high half-intensity temperatures of the glow curve at the ascending and descending side of the peak. We adopted a similar approach as earlier reported by Furetta et al (2000) for analyzing the TL glow curve where they did deconvolution based on Gaussian function and then analysed the individual peak using Chen's peak method using (1).

$$
E_{\alpha}=c_{\alpha}\left(\frac{k T_{\mathrm{m}}^{2}}{\alpha}\right)-b_{\alpha}\left(2 k T_{\mathrm{m}}\right),
$$

with $\alpha=\tau, \delta, \quad \omega ; \tau=T_{\mathrm{m}}-T_{1} ; \quad \delta=T_{2}-T_{1} ; \quad \omega=T_{2}-T_{1}$; $c_{\tau}=1.51+3.0 \quad\left(\mu_{\mathrm{g}}-0.42\right) ; c_{\delta}=0.976+7.3 \quad\left(\mu_{\mathrm{g}}-0.42\right)$; $c_{\omega}=2.52+10.2\left(\mu_{\mathrm{g}}-0.42\right) ; b_{\tau}=1.58+4.2\left(\mu_{\mathrm{g}}-0.42\right)$; $b_{\delta}=0 ; b_{\omega}=1$.

Once $E$ and $b$ are known, $s$ can be evaluated by (Chen and Kirsh 1981)

$$
\frac{\beta E}{k T_{\mathrm{m}}^{2}}=s \exp \left(-E / k T_{\mathrm{m}}\right)\left[1+(b-1) \Delta_{\mathrm{m}}\right]
$$

These parameters can be modified during the best fit procedure using glow curve deconvolution (GCD) program until the best fit is achieved which can be confirmed by the figure of merit (FOM). Some authors have reported similar studies for evaluation of kinetic parameters using Chen's peak method applied directly to the peaks which were deconvoluted using origin 6.1 software without using any GCD function (Nagabhushana et al 2008). In the present study we deconvoluted the TL glow curves based on Gaussian functions into four peaks which were also confirmed by the thermal cleaning method (Yazici and Haciibrah 2001). The isolated peaks were analyzed by Peak Chen method (Chen 1969) to evaluate the peak parameters using (1) and (2). The calculated parameters were used as initial parameters for the GCD basic function suggested by Kittis et al (1998) for the first, second and general order kinetics glow peaks given in (3)-(5) respectively.

First order

$$
\begin{aligned}
I(T)= & I_{\mathrm{m}} \exp \left[1+\left(\frac{E}{k T}\right) \frac{T-T_{\mathrm{m}}}{T_{\mathrm{m}}}-\left(\frac{T^{2}}{T_{\mathrm{m}}^{2}}\right)\right. \\
& \left.\exp \left\{\left(\frac{E}{k T}\right) \frac{T-T_{\mathrm{m}}}{T_{\mathrm{m}}}\right\}(1-\Delta)-\Delta_{\mathrm{m}}\right]
\end{aligned}
$$

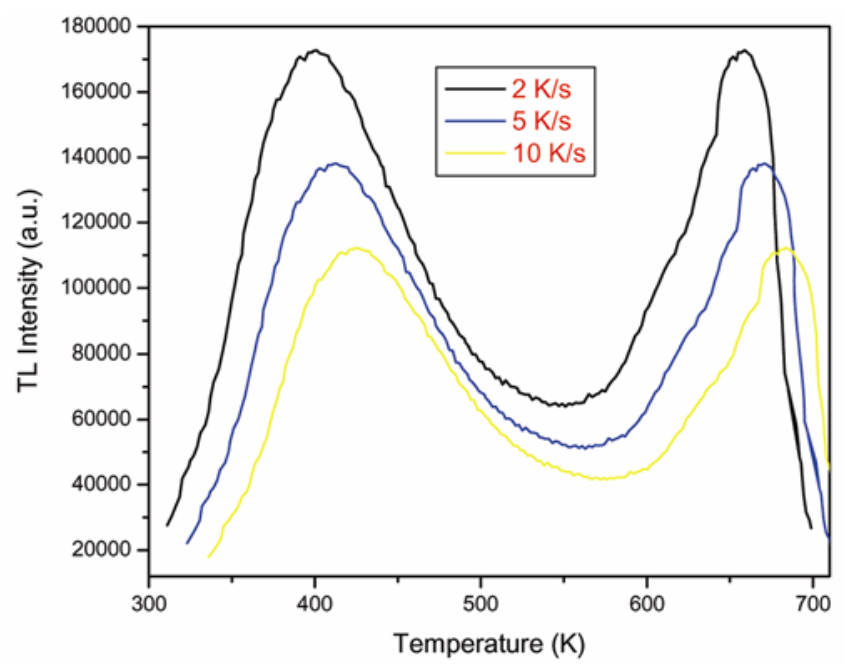

Figure 4. Effect of different heating rates on TL response of $\mathrm{BaS}: \mathrm{Bi}(0.4 \mathrm{~mol} \%)$. 
Table 1. Kinetic parameters using GCD function.

\begin{tabular}{lcccc}
\hline $\begin{array}{l}\text { Peak } \\
\text { number }\end{array}$ & $\begin{array}{c}T_{\max } \\
(\mathrm{K})\end{array}$ & $\begin{array}{c}\text { Order of } \\
\text { kinetics }(b)\end{array}$ & $\begin{array}{c}\text { Activation energy } \\
E(\mathrm{eV})\end{array}$ & $\begin{array}{c}\text { Frequency } \\
\text { factor }(\mathrm{s})\end{array}$ \\
\hline First peak & 395 & $2(0.47)$ & 0.35 & $1.05 \times 10^{6}$ \\
Second peak & 477 & $2(0.51)$ & 0.38 & $3.64 \times 10^{8}$ \\
Third peak & 626 & $2(0.52)$ & 1.20 & $1.38 \times 10^{9}$ \\
Fourth peak & 663 & $2(0.51)$ & 1.8 & $9.98 \times 10^{10}$ \\
\hline
\end{tabular}

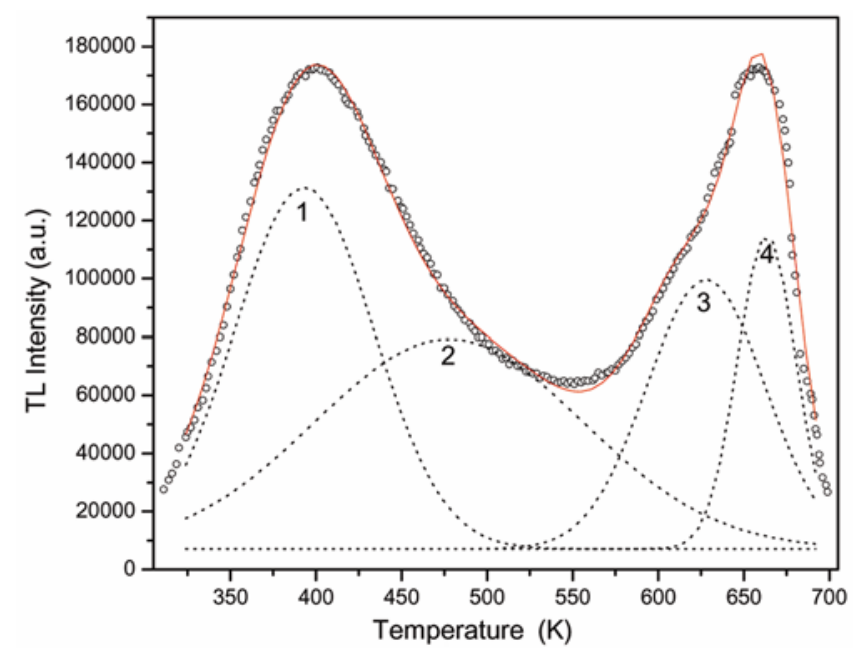

Figure 5. Comparison between theoretical (-) and experimental (-O-) fitted glow curve of $\mathrm{BaS}: \mathrm{Bi}$ exposed to gamma radiation. Deconvoluted single fitted glow curves $1,2,3,4(\cdots \cdots)$ are also shown

Second order

$$
\begin{aligned}
I(T)= & 4 I_{\mathrm{m}} \exp \left\{\left(\frac{E}{k T}\right) \frac{T-T_{\mathrm{m}}}{T_{\mathrm{m}}}\right\} \\
& {\left[\left(\frac{T^{2}}{T_{\mathrm{m}}^{2}}\right)(1-\Delta) \exp \left\{\left(\frac{E}{k T}\right) \frac{T-T_{\mathrm{m}}}{T_{\mathrm{m}}}\right\}+1+\Delta_{\mathrm{m}}\right]^{-2} . }
\end{aligned}
$$

General order

$$
\begin{aligned}
& I(T)=I_{\mathrm{m}} b^{\frac{b}{b-1}} \exp \left(\frac{E}{k T} \frac{\left(T-T_{\mathrm{m}}\right)}{T_{\mathrm{m}}}\right) \\
& {\left[(b-1)(1-\Delta) \frac{T^{2}}{T_{m}^{2}} \exp \left(\frac{E}{k T} \frac{T-T_{m}}{T_{m}}\right)+Z_{m}\right]^{\frac{-b}{b-1}},}
\end{aligned}
$$

where $I(T)$ is the TL intensity at temperature $T(K), I_{\mathrm{m}}$ the maximum peak intensity, $E$ the activation energy $(\mathrm{eV})$ and $k$ the Boltzmann's constant $\left(8.6 \times 10^{-5} \mathrm{eVK}^{-1}\right)$, $\Delta=2 \mathrm{kT} / \mathrm{E}, \Delta_{\mathrm{m}}=2 \mathrm{kT} \mathrm{m} / E$ and $Z_{\mathrm{m}}=1+(b-1) \Delta_{\mathrm{m}}$.
Figure 5 shows the experimental glow curve for $\mathrm{Bi}$ $(0.4 \mathrm{~mol} . \%)$ doped $\mathrm{BaS}$ nanostructures at heating rate of $2 \mathrm{~K} / \mathrm{s}$, which has been deconvoluted into four peaks using GCD function.

The position of the respective peaks, trap parameters and order of kinetics at heating rate of $2 \mathrm{~K} / \mathrm{s}$ is shown in table 1 .

\section{Conclusions}

We have synthesized $\mathrm{BaS}$ nanocrystalline phosphors activated by $\mathrm{Bi}$ ions. The thermo luminescence studies in $\mathrm{BaS}$ : Bi nanocrystalline phosphors irradiated with gamma rays are presented. The traps formed by gamma irradiation in nanomaterials are deeper than their bulk counterparts. The kinetic parameters for this sample at heating rate of $2 \mathrm{~K} / \mathrm{s}$ is calculated by Chen method and theoretical peak obtained by GCD function given by Kitis overlapped the experimental peak. It also has a linear response to a wide range of gamma doses, making it quiet suitable for TL dosimetry.

\section{Acknowledgements}

The authors are thankful to the Director of Inter University Accelerator Centre (IUAC), New Delhi for providing the experimental facilities. Financial support to $\mathrm{A} \mathrm{V}$ in the form of a Fellowship from IUAC, New Delhi through the project (UFUP) is gratefully acknowledged.

\section{References}

Akselrod M S, Larsen N, Whitley V and Mckeever S W S 1998 J. Appl. Phys. 842636

Chen R 1969 J. Appl. Phys. 40570

Chen R and Krish Y 1981 Anal. thermal stimulated process (New York: Pergamon)

Furetta C, Kitis G and Kuo C H Nucl. Instrum. Methods Phys. Res 2000 B160 65

Horowtz Y S, Avila O and Rodrigues M 2001 Nucl. Instrum. Methods Phys. Res. B184 85

Kitis G, Gomez-Ros J M and Tuyn J W N 1998 J. Phys. D Appl. Phys. 312636 
Lochab S P, Sahare P D, Chauhan R S, Salah N and Pandey A 2006 J. Phys. D: Appl. Phys. 391786

Mahajna S, Horowtz Y S 1997 J. Phys. D. Appl. Phys. 302603

Nagabhushana K R, Lakaminarasappa B N and Singh F 2008 Radiation Measurement 43651

Rao R P 1986 J. Mater. Sci. 213357

Sahare P D, Ranjan R, Salah N and Lochab S P 2007 J. Phys. D: Appl. Phys. 40759
Scherer P 1918 (Nachr. Ges. Wiss. Gottingen) pp. 96-100

Singh S, Vij A, Lochab S P, Ravi Kumar and Nafa Singh 2010 Mater. Res. Bull. 45523

Vij A, Lochab S P, Kumar R and Singh N 2009b J. Alloys Compounds

Vij A, Lochab S P, Singh S, Kumar R and Singh N 2009a J. Alloys Compounds 486554

Yazici N A and Haciibrah Y 2001 Turk J. Phys. 25249 